\title{
Covering-Based Rough Sets on Eulerian Matroids
}

\author{
Bin Yang, Ziqiong Lin, and William Zhu \\ Lab of Granular Computing, Minnan Normal University, Zhangzhou 363000, China \\ Correspondence should be addressed to William Zhu; williamfengzhu@gmail.com
}

Received 3 May 2013; Accepted 8 July 2013

Academic Editor: Zhihong Guan

Copyright (c) 2013 Bin Yang et al. This is an open access article distributed under the Creative Commons Attribution License, which permits unrestricted use, distribution, and reproduction in any medium, provided the original work is properly cited.

\begin{abstract}
Rough set theory is an efficient and essential tool for dealing with vagueness and granularity in information systems. Coveringbased rough set theory is proposed as a significant generalization of classical rough sets. Matroid theory is a vital structure with high applicability and borrows extensively from linear algebra and graph theory. In this paper, one type of covering-based approximations is studied from the viewpoint of Eulerian matroids. First, we explore the circuits of an Eulerian matroid from the perspective of coverings. Second, this type of covering-based approximations is represented by the circuits of Eulerian matroids. Moreover, the conditions under which the covering-based upper approximation operator is the closure operator of a matroid are presented. Finally, a matroidal structure of covering-based rough sets is constructed. These results show many potential connections between covering-based rough sets and matroids.
\end{abstract}

\section{Introduction}

Various theories and methods have been proposed to deal with incomplete and insufficient information in classification, concept formation, and data analysis in data mining. For example, fuzzy set theory [1-3], rough set theory [4, 5], computing with words $[6,7]$, and linguistic dynamic systems [8] have been developed and applied to real-world problems. Classical rough sets were originally proposed by Pawlak as a useful tool for dealing with the fuzzy and uncertain problems in information systems and have already been an efficient tool for data pre-process and widely used in fields such as process control, economics, medical diagnosis, conflict analysis, and other fields [9-12]. Covering-based rough set has been proposed as a generalization of classical rough set and the study on it is necessary and important. Particularly, with the fast development of computer science and technology in recent years, how to use the effective mathematical tools to solve practical problems has become more and more essential. Of course, as an efficient tool, the study on coveringbased rough sets is fetching in more and more researchers. Zhu [12] investigated some basic properties of covering-based rough sets and their comparisons with the corresponding ones of classical rough sets. Zhang et al. [10] investigated three types of covering-based rough sets with an axiomatic approach. In addition, some detailed works of covering-based rough sets can be found in the literatures [13-17]. Therefore, there is profound theoretical and practical significance to study the covering-based rough sets.

The concept of matroids was originally introduced by Whitney [18] in 1935 as a generalization of graph theory and linear algebra. Matroid theory is a structure that generalizes linear independence in vector spaces and has a variety of applications in many fields such as algorithm design [19] and combinatorial optimization [20]. In theory, matroid theory provides a good platform to connect it with other theories. Some interesting results about the connection between matroid theory and rough set theory can be found in the literatures [21-25].

In this paper, through Eulerian matroids, one type of covering-based approximation operators is represented through the perspective of Eulerian matroids. First, since the family of circuits of an Eulerian matroid is a covering, then the properties, such as irreducible, semireduced, and minimal description, are investigated from the viewpoint of coverings. Second, the second type of covering-based upper and lower approximation operators is represented by the circuits of the Eulerian matroid. In fact, some types of covering-based lower approximation operators are equal, and they can be represented by the circuits of the restriction matroids of Eulerian 
matroids. Moreover, we obtain the condition under which the covering-based upper approximation operator is the closure operator of a matroid. Finally, a matroidal structure of covering-based rough sets is constructed by a type of covering with some special properties.

The remainder of this paper is organized as follows. In Section 2, some basic concepts and properties related to covering-based rough sets and matroids are introduced. In Section 3, some properties of the covering induced by an Eulerian matroid are investigated. Moreover, the focus here is the covering-based approximation operators denoted by the circuits of Eulerian matroids. In Section 4, a matroidal structure of covering-based rough sets is constructed by a type of covering with some special properties. Section 5 concludes this paper.

\section{Preliminaries}

In this section, we review some fundamental definitions and results of covering-based rough sets and matroids.

2.1. Covering-Based Rough Sets. In this subsection, we recall some basic definitions and results of covering-based rough sets used in this paper. For detailed descriptions about covering-based rough sets, please refer to $[9,11,12,26]$.

Definition 1 (covering [11]). Let $U$ be a universe of discourse, $\mathrm{C}$ a family of subsets of $U$. If none of subsets in $\mathrm{C}$ is empty, and $\cup \mathbf{C}=U$, then $\mathbf{C}$ is called a covering of $U$.

It is clear that a partition of $U$ is certainly a covering of $U$, so the concept of a covering is an extension of the concept of a partition. In the following discussion, the universe of discourse $U$ is considered to be finite.

Definition 2 (covering-based approximation space [11]). Let $U$ be a universe of discourse and $\mathbf{C}$ a covering of $U$. The ordered pair $\langle U, \mathbf{C}\rangle$ is called a covering-based approximation space.

Definition 3 (minimal description [11]). Letting $\langle U, \mathbf{C}\rangle$ be a covering-based approximation space, $x \in U$, then set family

$$
\begin{aligned}
& M d_{\mathbf{C}}(x) \\
& \quad=\{K \in \mathbf{C} \mid x \in K \wedge(\forall S \in \mathbf{C} \wedge x \in S \wedge S \subseteq K \Longrightarrow K=S)\}
\end{aligned}
$$

is called the minimal description of $x$.

Definition 4 (unary covering [12]). Let $\mathbf{C}$ be a covering of $U$. $\mathrm{C}$ is called unary if $\forall x \in U,\left|M d_{\mathrm{C}}(x)\right|=1$.

Definition 5 (representative element [12]). Let $\mathbf{C}$ be a covering of $U$. If $x \in K \in \mathrm{C}$ satisfies the following condition:

$$
\forall K^{\prime} \in \mathbf{C} \quad\left(x \in K^{\prime} \Longrightarrow K \subseteq K^{\prime}\right),
$$

then $x$ is called a representative element of $K$.

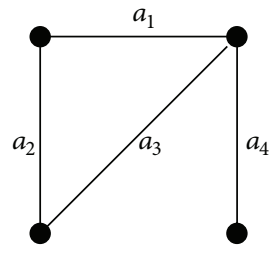

Figure 1: A graph.

Definition 6 (representative covering [12]). Let $\mathbf{C}$ be a covering of $U$. If $\forall K \in \mathbf{C}, K$ has a representative element, one says $\mathrm{C}$ is representative.

Definition 7 (exact covering [12]). Let $\mathbf{C}$ be a covering of $U$. If $\forall \mathbf{C}^{\prime} \subseteq \mathbf{C}$,

$$
\left\{K \in \mathbf{C} \mid K \subseteq \bigcup \mathbf{C}^{\prime}\right\}=\left\{K \in \mathbf{C} \mid \exists K^{\prime} \in \mathbf{C}^{\prime}\left(K \subseteq K^{\prime}\right)\right\},
$$

one says the covering $\mathbf{C}$ is exact.

The following lemma shows that the concepts of representative and exact coverings are equivalent.

Lemma 8 (see [12]). Let $\mathbf{C}$ be a covering of $U$. C is representative if and only if $\mathbf{C}$ is exact.

2.2. Matroids. Matroid theory was established as a generalization of graph theory and linear algebra. This theory was used to study abstract relations on a subset, and it used both of these areas of mathematics for its motivation, its basic examples, and its notation. With the rapid development of matroid theory in recent years, it has already become an effective mathematical tool to study other mathematic branches. In this subsection, we recall some definitions, examples, and results of matroids.

Definition 9 (matroid [27]). A matroid is an ordered pair $M=$ $(U, \mathscr{F})$, where $U$ is a finite set and $\mathscr{I}$ a family of subsets of $U$ with the following three properties:

(I1) $\emptyset \in \mathscr{I}$;

(I2) if $I \in \mathscr{I}$, and $I^{\prime} \subseteq I$, then $I^{\prime} \in \mathscr{I}$;

(I3) if $I_{1}, I_{2} \in \mathscr{I}$, and $\left|I_{1}\right|<\left|I_{2}\right|$, then there exists $e \in$ $I_{2}-I_{1}$ such that $I_{1} \bigcup\{e\} \in \mathscr{I}$, where $|I|$ denotes the cardinality of $I$.

Any element of $\mathscr{I}$ is called an independent set.

Example 10. Let $G=(V, U)$ be the graph as shown in Figure 1 . Denote $\mathscr{I}=\{I \subseteq U \mid I$ does not contain a cycle of $G\}$, that is, $\mathscr{I}=\left\{\emptyset,\left\{a_{1}\right\},\left\{a_{2}\right\},\left\{a_{3}\right\},\left\{a_{4}\right\},\left\{a_{1}, a_{2}\right\},\left\{a_{1}, a_{3}\right\},\left\{a_{1}, a_{4}\right\}\right.$, $\left.\left\{a_{2}, a_{3}\right\},\left\{a_{2}, a_{4}\right\},\left\{a_{3}, a_{4}\right\},\left\{a_{1}, a_{2}, a_{4}\right\},\left\{a_{1}, a_{3}, a_{4}\right\},\left\{a_{2}, a_{3}, a_{4}\right\}\right\}$.

Then $M=(U, \mathscr{I})$ is a matroid, where $U=\left\{a_{1}, a_{2}, a_{3}, a_{4}\right\}$.

In fact, if a subset is not an independent set, then it is a dependent set of the matroid. In other words, the dependent set of a matroid generalizes the cycle in graphs. Based on the dependent set, we introduce other concepts of a matroid. 
For this purpose, several denotations are presented in the following definition.

Definition 11 (see [27]). Let $\mathscr{A}$ be a family of subsets of $U$. One can denote

$$
\begin{aligned}
& \operatorname{Max}(\mathscr{A})=\{X \in \mathscr{A} \mid \forall Y \in \mathscr{A}, X \subseteq Y \Rightarrow X=Y\}, \\
& \operatorname{Min}(\mathscr{A})=\{X \in \mathscr{A} \mid \forall Y \in \mathscr{A}, Y \subseteq X \Rightarrow X=Y\}, \\
& \operatorname{Opp}(\mathscr{A})=\{X \subseteq U \mid X \notin \mathscr{A}\}, \\
& \operatorname{Com}(\mathscr{A})=\{X \subseteq U \mid \sim X \in \mathscr{A}\}, \text { where } \sim X=U-X .
\end{aligned}
$$

Definition 12 (base [27]). Let $M=(U, \mathscr{I})$ be a matroid. A maximal independent set in $M$ is called a base of $M$, and one denotes the family of all bases of $M$ by $\mathscr{B}(M)$, that is, $\mathscr{B}(M)=$ $\operatorname{Max}(\mathscr{I})$.

Example 13 (continued from Example 10). According to Definition 12, we have $\mathscr{B}(M)=\left\{\left\{a_{1}, a_{2}, a_{4}\right\},\left\{a_{1}, a_{3}, a_{4}\right\}\right.$, $\left.\left\{a_{2}, a_{3}, a_{4}\right\}\right\}$.

A matroid and its bases are uniquely determined by each other. In other words, a matroid can also be defined from the viewpoint of the base.

Theorem 14 (base axiom [27]). Let $\mathscr{B}$ be a family of subsets of $U$. Then there exists $M=(U, \mathscr{I})$ such that $\mathscr{B}=\mathscr{B}(M)$ if and only if $\mathscr{B}$ satisfies the following two conditions:

(B1) $\mathscr{B} \neq \emptyset$;

(B2) if $B_{1}, B_{2} \in \mathscr{B}$ and $x \in B_{1}-B_{2}$, then there exists $y \in$ $B_{2}-B_{1}$ such that $\left(B_{1}-\{x\}\right) \bigcup\{y\} \in \mathscr{B}$.

Definition 15 (circuit [27]). Let $M=(U, \mathscr{I})$ be a matroid. A minimal dependent set in $M$ is called a circuit of $M$, and one denotes the family of all circuits of $M$ by $\mathscr{C}(M)$, that is,

$$
\mathscr{C}(M)=\operatorname{Min}(\operatorname{Opp}(\mathscr{I})) .
$$

Example 16 (continued from Example 10). According to Definition 15, we have $\mathscr{C}(M)=\left\{\left\{a_{1}, a_{2}, a_{3}\right\}\right\}$.

Theorem 17 (circuit axiom [27]). Let $M=(U, \mathscr{I})$ be a matroid and $\mathscr{C}=\mathscr{C}(M)$. Then $\mathscr{C}$ satisfies the following three properties:

(C1) $\emptyset \notin \mathscr{C}$;

(C2) if $C_{1}, C_{2} \in \mathscr{C}$ and $C_{1} \subseteq C_{2}$, then $C_{1}=C_{2}$;

(C3) if $C_{1}, C_{2} \in \mathscr{C}, C_{1} \neq C_{2}$ and $e \in C_{1} \cap C_{2}$, then there exists $C_{3} \in \mathscr{C}$ such that $C_{3} \subseteq\left(C_{1} \cup C_{2}\right)-\{e\}$.

A matroid uniquely determines its circuits and vice versa. The following theorem shows that a matroid can be defined from the viewpoint of circuits.

Theorem 18 (see [27]). Let $U$ be a nonempty and finite set and $\mathscr{C}$ a family of subsets of $U$. If $\mathscr{C}$ satisfies (C1), (C2), and (C3) of Theorem 17, then there exists $M=(U, \mathscr{I})$ such that $\mathscr{C}=$ $\mathscr{C}(M)$.

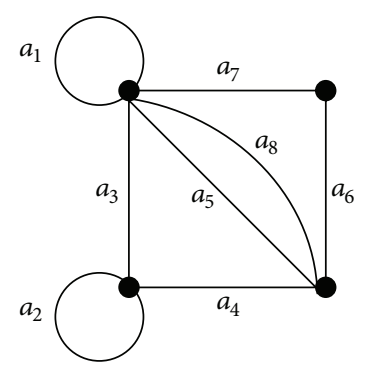

Figure 2: A graph.

Definition 19 (rank function [27]). Let $M=(U, \mathscr{I})$ be a matroid. One can define the rank function of $M$ as follows: for all $X \in 2^{U}$,

$$
r_{M}(X)=\max \{|I| \mid I \subseteq X, I \in \mathscr{I}\} .
$$

$r_{M}(X)$ is calledthe rank of $X$ in $M$.

Based on the rank function of a matroid, one can define the closure operator, which reflects the dependency between a set and elements.

Definition 20 (closure [27]). Let $M=(U, \mathscr{I})$ be a matroid. The closure operator $\mathrm{cl}_{M}$ of $M$ is defined as $\mathrm{cl}_{M}(X)=\{e \epsilon$ $\left.U \mid r_{M}(X \bigcup\{e\})=r_{M}(X)\right\}$ for all $X \in 2^{U} \cdot \operatorname{cl}_{M}(X)$ is called the closure of $X$ in $M$.

\section{Covering-Based Rough Sets on Eulerian Matroids}

In this section, we provide equivalent formulations of some important concepts and properties of covering-based rough sets from the viewpoint of Eulerian matroids. Specifically, the covering-based upper and lower approximation operators are characterized by the circuits of matroids. For this purpose, some definitions and properties of the covering induced by an Eulerian matroid are presented.

The following definition introduces Eulerian matroids, which is a special kind of matroids, and has sound theoretical foundations and wide applications.

Definition 21 (Eulerian matroid [27]). Let $M=(U, \mathscr{I})$ be a matroid. If there exist $C_{1}, C_{2}, \ldots, C_{m} \in \mathscr{C}(M)$ such that $C_{i} \cap C_{j}=\emptyset(i \neq j, i, j=1,2, \ldots, m)$ and $U=\bigcup_{i=1}^{m} C_{i}$, then one says $M$ is an Eulerian matroid.

Example 22. Let $G=(V, U)$ be the graph as shown in Figure 2. Denote $\mathscr{C}=\{C \subseteq U \mid C$ does a cycle of $G\}$, that is, $\mathscr{C}=$ $\left\{\left\{a_{1}\right\},\left\{a_{2}\right\},\left\{a_{5}, a_{8}\right\},\left\{a_{3}, a_{4}, a_{5}\right\},\left\{a_{3}, a_{4}, a_{8}\right\},\left\{a_{5}, a_{6}, a_{7}\right\},\left\{a_{6}, a_{7}\right.\right.$, $\left.\left.a_{8}\right\},\left\{a_{3}, a_{4}, a_{6}, a_{7}\right\}\right\}$. Then there exists a matroid $M=(U, \mathscr{F})$ such that $\mathscr{C}=\mathscr{C}(M)$, where $U=\left\{a_{1}, a_{2}, a_{3}, a_{4}, a_{5}, a_{6}, a_{7}, a_{8}\right\}$. Clearly, $M$ is an Eulerian matroid.

According to the above definition, it is clear that $\mathscr{C}(M)$ is a covering of $U$. In other words, we construct a covering by an Eulerian matroid. 
Example 23 (continued from Example 22). It is easy to check that the set family $\mathscr{C}=\left\{\left\{a_{1}\right\},\left\{a_{2}\right\},\left\{a_{5}, a_{8}\right\},\left\{a_{3}, a_{4}, a_{5}\right\}\right.$, $\left.\left\{a_{3}, a_{4}, a_{8}\right\},\left\{a_{5}, a_{6}, a_{7}\right\},\left\{a_{6}, a_{7}, a_{8}\right\},\left\{a_{3}, a_{4}, a_{6}, a_{7}\right\}\right\}$ is a covering of $U$.

Proposition 24. Let $M=(U, \mathscr{I})$ be an Eulerian matroid and $x \in U$. Then

$$
M d_{\mathscr{C}(M)}(x)=\{C \in \mathscr{C}(M) \mid x \in C\} .
$$

Proof. It is obvious $M d_{\mathscr{C}(M)}(x) \subseteq\{C \in \mathscr{C}(M) \mid x \in C\}$. Suppose $M d_{\mathscr{C}(M)}(x) \neq\{C \in \mathscr{C}(M) \mid x \in C\}$. Hence $\{C \in$ $\mathscr{C}(M) \mid x \in C\}-M d_{\mathscr{C}(M)}(x) \neq \emptyset$. Suppose $K \in\{C \in \mathscr{C}(M) \mid x \in$ $C\}-M d_{\mathscr{C}(M)}(x)$. Then there exists $L \in \mathscr{C}(M)$ such that $x \in L$ and $L \subset K$. It is contradiction to (C2) of Theorem 17.

Example 25 (continued from Example 22). We have

$$
\begin{aligned}
M d_{\mathscr{C}(M)}\left(a_{1}\right) & =\left\{\left\{a_{1}\right\}\right\}, \quad M d_{\mathscr{C}(M)}\left(a_{2}\right)=\left\{\left\{a_{2}\right\}\right\}, \\
M d_{\mathscr{C}(M)}\left(a_{5}\right) & =\left\{\left\{a_{5}, a_{8}\right\},\left\{a_{3}, a_{4}, a_{5}\right\},\left\{a_{5}, a_{6}, a_{7}\right\}\right\}, \\
M d_{\mathscr{C}(M)}\left(a_{8}\right) & =\left\{\left\{a_{5}, a_{8}\right\},\left\{a_{3}, a_{4}, a_{8}\right\},\left\{a_{6}, a_{7}, a_{8}\right\}\right\}, \\
M d_{\mathscr{C}(M)}\left(a_{3}\right) & =\left\{\left\{a_{3}, a_{4}, a_{5}\right\},\left\{a_{3}, a_{4}, a_{8}\right\},\left\{a_{3}, a_{4}, a_{6}, a_{7}\right\}\right\} \\
= & M d_{\mathscr{C}(M)}\left(a_{4}\right), \\
M d_{\mathscr{C}(M)}\left(a_{6}\right)= & \left\{\left\{a_{5}, a_{6}, a_{7}\right\},\left\{a_{6}, a_{7}, a_{8}\right\},\left\{a_{3}, a_{4}, a_{6}, a_{7}\right\}\right\} \\
= & M d_{\mathscr{C}(M)}\left(a_{7}\right) .
\end{aligned}
$$

In fact, the concepts of unary covering, representative covering, and exact covering play a vital role in coveringbased rough sets. Based on the properties of the Eulerian matroid and the above proposition, the following remark is presented.

Remark 26. If $M=(U, \mathscr{I})$ is an Eulerian matroid, then $\mathscr{C}(M)$ is not a representative covering. In other words, $\mathscr{C}(M)$ is not an exact covering.

Example 27 (continued from Example 22). It is easy to know $\mathscr{C}=\left\{\left\{a_{1}\right\},\left\{a_{2}\right\},\left\{a_{5}, a_{8}\right\},\left\{a_{3}, a_{4}, a_{5}\right\},\left\{a_{3}, a_{4}, a_{8}\right\},\left\{a_{5}, a_{6}, a_{7}\right\},\left\{a_{6}\right.\right.$, $\left.\left.a_{7}, a_{8}\right\},\left\{a_{3}, a_{4}, a_{6}, a_{7}\right\}\right\}$ is not a representative and exact covering of $U$.

Proposition 28. Let $M=(U, \mathscr{I})$ be an Eulerian matroid. Then $\mathscr{C}(M)$ is a unary covering if and only if $\mathscr{C}(M)$ is a partition of $U$.

Proof. $(\Rightarrow)$ : Let $\mathscr{C}(M)$ be a unary covering of $U$ and $x \in U$. Then $\left|M d_{\mathscr{C}(M)}(x)\right|=1$. If $\mathscr{C}(M)$ is not a partition of $U$, then there exists $x \in U$ such that there exist $C_{1}, C_{2} \in \mathscr{C}(M)$, $C_{1} \neq C_{2}$ and $x \in C_{1} \cap C_{2}$, that is, $\left|M d_{\mathscr{C}(M)}(x)\right| \geq 2$. It is contradictory.

$(\Leftarrow)$ : It is straightforward.

Example 29 (continued from Example 22). Clearly, according to Proposition 28, since $\mathscr{C}(M)=\left\{\left\{a_{1}\right\},\left\{a_{2}\right\},\left\{a_{5}, a_{8}\right\},\left\{a_{3}, a_{4}\right.\right.$, $\left.\left.a_{5}\right\},\left\{a_{3}, a_{4}, a_{8}\right\},\left\{a_{5}, a_{6}, a_{7}\right\},\left\{a_{6}, a_{7}, a_{8}\right\},\left\{a_{3}, a_{4}, a_{6}, a_{7}\right\}\right\}$ is not a partition of $U$, then $\mathscr{C}(M)$ is not a unary covering of $U$.

In covering-based rough sets, a pair of covering-based approximation operators are used to describe an object. In the following definition, a widely used pair of covering-based approximation operators are introduced.

Definition 30 (covering-based approximation set family [12]). Let $\mathbf{C}$ be a covering of $U$. For any set $X \subseteq U$, set families

$$
\begin{gathered}
\underline{\mathbf{C}}(X)=\{K \in \mathbf{C} \mid K \subseteq X\}, \\
\overline{\mathbf{C}}(X)=\{K \in \mathbf{C} \mid K \bigcap X \neq \emptyset\}
\end{gathered}
$$

are called the covering-based lower and upper approximation set families of $X$, respectively.

Definition 31 (covering-based approximation operator [12]). Let $\mathbf{C}$ be a covering of $U$. For any set $X \subseteq U$,

$$
\underline{X}=\bigcup \underline{\mathbf{C}}(X), \quad \bar{X}=\bigcup \overline{\mathbf{C}}(X)
$$

are called the covering-based lower and upper approximations of $X$, respectively.

Definition 32 (restriction matroid [27]). Let $M=(U, \mathscr{I})$ be a matroid and $X \subseteq U$. Suppose $\mathscr{I}_{X}=\{I \subseteq X \mid I \in \mathscr{I}\}$. Then $\left(X, \mathscr{I}_{X}\right)$ is a matroid and called the restriction matroid of $X$ in $M$. One denotes the restriction matroid of $X$ in $M$ as $M \mid X$, that is, $M \mid X=\left(X, \mathscr{I}_{X}\right)$.

In the following proposition, a widely used pair of covering-based approximation operators are represented by the circuits of Eulerian matroids.

Proposition 33. Let $M=(U, \mathscr{I})$ be an Eulerian matroid and $X \subseteq U$. Then

$$
\begin{aligned}
& \overline{\mathscr{C}(M)}(X)=\mathscr{C}(M \mid X), \\
& \overline{\mathscr{C}(M)}(X)=\{C \in \mathscr{C}(M) \mid C \bigcap X \neq \emptyset\} .
\end{aligned}
$$

Proof. Let $\mathscr{C}_{1}=\{C \in \mathscr{C}(M) \mid C \subseteq X\}$. According to Definition 30 , we need to prove only $\mathscr{C}_{1}=\mathscr{C}(M \mid X)$. On one hand, for any $C \in \mathscr{C}_{1}$, we have $C \subseteq X$ and $C \notin \mathscr{I}_{X}$. Since $C \in \mathscr{C}(M)$, then, for any $e \in C, C-\{e\} \in \mathscr{I}$ and $C-\{e\} \subseteq X$ hold. Hence, $C-\{e\} \in \mathscr{I}_{X}$, that is, $C \in \mathscr{C}(M \mid X)$ and $\mathscr{C}_{1} \subseteq \mathscr{C}(M \mid X)$. On the other hand, for any $C^{\prime} \in \mathscr{C}(M \mid X)$ and any $e \in C^{\prime}$, it is easy to prove $C^{\prime}-\{e\} \in \mathscr{I}_{X} \subseteq \mathscr{I}$. Since $C^{\prime} \subseteq X$ and $C^{\prime} \notin \mathscr{I}_{X}$, then $C^{\prime} \notin \mathscr{I}$. Therefore, $C^{\prime} \in \mathscr{C}(M)$. Since $C^{\prime} \subseteq X$, then $\mathscr{C}(M \mid X) \subseteq \mathscr{C}_{1}$ holds.

Example 34. Let $M=(U, \mathscr{I})$ be a matroid, where $U=\{a$, $b, c, d, e, f\}$ and $\mathscr{C}(M)=\{\{e, f\},\{a, d, e\},\{a, d, f\},\{b, c, e\}$, $\{b, c, f\},\{a, b, c, d\}\}$. Clearly, $M$ is an Eulerian matroid and 
$\mathscr{C}(M)$ a covering of $U$. Suppose $X_{1}=\{a, b, d, f\}, X_{2}=$ $\{a, b, c, e, f\}$, and $X_{3}=\{d\}$. Then

$$
\begin{aligned}
& \frac{\mathscr{C}(M)}{\overline{\mathscr{C}(M)}}\left(X_{1}\right)=\mathscr{C}\left(M \mid X_{1}\right)=\mathscr{C}(M), \\
& \overline{\mathscr{C}(M)}\left(X_{2}\right)=\mathscr{C}\left(M \mid X_{2}\right)=\{\{e, f\},\{b, c, e\},\{b, c, f\}\}, \\
& \overline{\mathscr{C}(M)}\left(X_{2}\right)=\mathscr{C}(M), \\
& \overline{\mathscr{C}(M)}\left(X_{3}\right)=\mathscr{C}\left(M \mid X_{3}\right)=\emptyset, \\
& \overline{\mathscr{C}(M)}\left(X_{3}\right)=\{\{a, d, e\},\{a, d, f\},\{a, b, c, d\}\} .
\end{aligned}
$$

Similarly, according to the above proposition, we can obtain the following proposition.

Proposition 35. Let $M=(U, \mathscr{I})$ be an Eulerian matroid and $X \subseteq U$. Then

$$
\begin{gathered}
\underline{X}=\bigcup \mathscr{C}(M \mid X), \\
\bar{X}=\bigcup\{C \in \mathscr{C}(M) \mid C \bigcap X \neq \emptyset\} .
\end{gathered}
$$

Proof. It is easy to prove this proposition by Definition 31 and Proposition 33.

Example 36 (continued from Example 34). As shown in Example 34, the covering-based lower and upper approximation sets of $X_{1}, X_{2}$, and $X_{3}$, respectively, are $\underline{X}_{1}=\{a, d, f\}$, $\bar{X}_{1}=\bigcup \mathscr{C}(M)=U, \underline{X}_{2}=\bigcup \mathscr{C}\left(M \mid X_{2}\right)=\{b, c, e, f\}, \bar{X}_{2}=$ $\bigcup \mathscr{C}(M)=U, \underline{X}_{3}=\bigcup \mathscr{C}\left(M \mid X_{3}\right)=\emptyset, \bar{X}_{3}=\bigcup \mathscr{C}(M)=U$.

The above proposition shows that the covering-based lower and covering-based upper approximation operators of covering-based rough sets can be denoted by the circuits of matroids. Li and Liu [21] have already proved the closure operator of matroids and covering-based upper approximation operator are equivalent if and only if the covering is unary. Based on their works, the following proposition can be presented.

Proposition 37. Let $M=(U, \mathscr{I})$ be an Eulerian matroid. For any $X \subseteq U, \bar{X}=c l_{M}(X)$ if and only if $\mathscr{C}(M)$ is a partition of $U$.

Proof. According to Proposition 28, $\mathscr{C}(M)$ is a unary covering if and only if $\mathscr{C}(M)$ is a partition of $U$. Hence, this proposition is easy to prove.

Example 38. Let $M=(U, \mathscr{I})$ be an Eulerian matroid, where $U=\{a, b, c, d, e, f\}$ and $\mathscr{C}(M)=\{\{a, b\},\{c, f\},\{d, e\}\}$. Suppose $X_{1}=\{a, b, e\}, X_{2}=\{c, d\}$, and $X_{3}=\{f\}$. Then $\bar{X}_{1}=$ $\{a, b, d, e\}, \bar{X}_{2}=\{c, d, e, f\}$, and $\bar{X}_{3}=\{c, f\}$, and $\mathrm{cl}_{M}\left(X_{1}\right)=$ $\{a, b, d, e\}, \operatorname{cl}_{M}\left(X_{2}\right)=\{c, d, e, f\}$ and $\mathrm{cl}_{M}\left(X_{3}\right)=\{c, f\}$.

Proposition 39. Let $M=(U, \mathscr{J})$ be an Eulerian matroid. For any $X \subseteq U, X \in \mathscr{I}$ if and only if $\overline{\mathscr{C}(M)}(\sim X)=\mathscr{C}(M)$.
Proof. $(\Rightarrow)$ : Suppose $X \in \mathscr{I}$. Clearly, it is easy to see $\overline{\mathscr{C}(M)}(\sim$ $X) \subseteq \mathscr{C}(M)$ according to Proposition 33. If there exists $C^{\prime} \in$ $\mathscr{C}(M)$ such that $C^{\prime} \notin \overline{\mathscr{C}(M)}(\sim X)$, then $C^{\prime} \cap(\sim X)=\emptyset$. Thus $C^{\prime} \subseteq X$. Clearly, it is a contradiction to $X \in \mathscr{F}(M)$. Then $C \in$ $\overline{\mathscr{C}(M)}(\sim X)$ for any $C \in \mathscr{C}(M)$, that is, $\mathscr{C}(M) \subseteq \overline{\mathscr{C}(M)}(\sim X)$. Hence $\overline{\mathscr{C}(M)}(\sim X)=\mathscr{C}(M)$.

$(\Leftarrow)$ : Suppose $X \notin \mathscr{I}$. Then there exists $C \in \mathscr{C}(M)$ such that $C \subseteq X$, that is, $C \bigcap(\sim X)=\emptyset$. Thus $C \notin \overline{\mathscr{C}(M)}(\sim X)$. Hence $\overline{\mathscr{C}(M)}(\sim X) \neq \mathscr{C}(M)$; it is a contradiction to $\overline{\mathscr{C}(M)}(\sim$ $X)=\mathscr{C}(M)$. Then $X \in \mathscr{I}$.

In fact, the above proposition indicates that the independent sets of an Eulerian matroid can be judged from the viewpoint of covering-based rough sets.

Example 40 (continued from Example 38). Since $\overline{\mathscr{C}(M)}(\sim$ $\left.X_{1}\right)=\{\{c, f\},\{d, e\}\}, \overline{\mathscr{C}(M)}\left(\sim X_{2}\right)=\{\{a, b\},\{c, f\},\{d, e\}\}$ and $\overline{\mathscr{C}(M)}\left(\sim X_{3}\right)=\{\{a, b\},\{c, f\},\{d, e\}\}$, then $X_{1} \notin \mathscr{I}, X_{2} \in \mathscr{I}$, and $X_{3} \in \mathscr{I}$.

In fact, it is easy to find that the circuits of an Eulerian matroid contain a partition of the universe at least. Therefore, we study the relationships between the circuits of an Eulerian matroid and its subset family.

Proposition 41. Let $M=(U, \mathscr{I})$ be an Eulerian matroid and $X \subseteq U$. Then there exists a partition $U / R=\left\{C_{1}, C_{2}, \ldots, C_{m}\right\} \subseteq$ $\mathscr{C}(M)$ such that $\underline{R}(X) \subseteq \underline{X}$ and $\bar{R}(X) \subseteq \bar{X}$, where $\underline{R}, \bar{R}$ are the classical approximation operators.

Proof. It is easy to prove this proposition by Definition 31 and Proposition 35.

Example 42 (continued from Example 38). It is not difficult to find that $U / R=\mathscr{C}(M)$. Hence, the covering-based upper and lower approximation operators are the classical upper and lower approximation operators, respectively.

Proposition 43. Let $M=(U, \mathscr{I})$ be an Eulerian matroid and $X \subseteq U$. Then $\overline{\mathscr{C}(M)}(X)=\mathscr{C}(M)(X)$ if and only if $\{\{x\} \mid x \in$ $X\} \subseteq \mathscr{C}(M)$.

Proof. $(\Rightarrow)$ : Suppose there exists $x^{\prime} \in X$ such that $\left\{x^{\prime}\right\} \notin$ $\mathscr{C}(M)$. Then there exists $C^{\prime}=\left\{x^{\prime}, x_{1}, \ldots, x_{s}\right\}(s \geq 1)$ such that $C^{\prime} \in \mathscr{C}(M)$ and $C^{\prime} \in \overline{\mathscr{C}(M)}\left(\left\{x^{\prime}\right\}\right)$. Since $\left\{x^{\prime}\right\} \subset C^{\prime}$, then $C^{\prime} \notin \mathscr{C}(M)\left(\left\{x^{\prime}\right\}\right)$. It is contradictory. Thus $\{\{x\} \mid x \in X\} \subseteq$ $\mathscr{C}(M)$.

$(\Leftarrow)$ : Since $\{\{x\} \mid x \in X\} \subseteq \mathscr{C}(M)$ holds for any $X \subseteq U$, then $\overline{\mathscr{C}(M)}(X)=\{\{x\} \mid x \in X\}=\mathscr{\mathscr { C }}(M)(X)$.

Similarly, according to the above proposition, the following proposition can be presented.

Proposition 44. Let $M=(U, \mathscr{I})$ be an Eulerian matroid and $X \subseteq U$. Then $\bar{X}=\underline{X}$ if and only if $\{\{x\} \mid x \in X\} \subseteq \mathscr{C}(M)$.

Since a family of circuits determines only one matroid, then the relationship between two coverings, which are 
induced by two Eulerian matroids, is presented in the following proposition.

Proposition 45. Let $M_{1}=\left(U, \mathscr{I}_{1}\right)$ and $M_{2}=\left(U, \mathscr{I}_{2}\right)$ be two Eulerian matroids. If $\mathscr{I}_{1} \neq \mathscr{I}_{2}$, then there exists $X \subseteq U$ such that $\overline{\mathscr{C}\left(M_{1}\right)}(X) \neq \overline{\mathscr{C}\left(M_{2}\right)}(X)$ and $\mathscr{C}\left(M_{1}\right)(X) \neq \mathscr{C}\left(M_{2}\right)(X)$.

In fact, the above proposition shows that different Eulerian matroids induce different covering-based approximation spaces and different covering-based approximation operators.

The above results show that many important concepts and properties of covering-based rough sets can be concisely characterized by corresponding concepts and properties in matroids. Therefore, covering-based rough sets may be efficient to study matroids.

\section{The Eulerian Matroid Induced by a Covering}

From the above definitions and properties, we know that the circuits of an Eulerian matroid can construct only one covering-based approximation space. Now, in this section, we wonder if a covering $\mathbf{C}$ of $U$ forms the family of circuits of an Eulerian matroid, what conditions should the covering C satisfies? For this purpose, the concepts of antichain and blocker are introduced.

Definition 46 (antichain [27]). Let $\mathscr{A}$ be a family of subsets of $U$. If, for any $A_{1}, A_{2} \in \mathscr{A}, A_{1} \nsubseteq A_{2}$ and $A_{2} \nsubseteq A_{1}$ hold, then one says $\mathscr{A}$ is an antichain of $U$.

According to the above definition, it is easy to see that the family of circuits and the family of bases of a matroid are all antichains.

Definition 47 (blocker [27]). Let $\mathscr{A}$ be an antichain of $U$. Then the blocker $b(\mathscr{A})$ of $\mathscr{A}$ can be denoted as follows:

$$
b(\mathscr{A})=\operatorname{Min}\{X \subseteq U \mid \forall A \in \mathscr{A}, X \bigcap A \neq \emptyset\} .
$$

Example 48. Let $\mathscr{A}=\{\{a, b\},\{a, c\},\{b, c, d\}\}$ be a family of subsets of $U=\{a, b, c, d\}$. Clearly, $\mathscr{A}$ is an antichain of $U$. Suppose $b(\mathscr{A})$ is the blocker of $\mathscr{A}$. Then $b(\mathscr{A})=$ $\operatorname{Min}\{\{a, b\},\{a, c\},\{a, d\},\{b, c\},\{a, b, c\},\{a, b, d\},\{a, c, d\},\{b, c$, $d\},\{a, b, c, d\}\}=\{\{a, b\},\{a, c\},\{a, d\},\{b, c\}\}$.

Theorem 49 (see [27]). Let $M(U, \mathscr{I})$ be a matroid. Then there exists another matroid on $U$, whose family of all bases is equal to $\operatorname{Com}(\mathscr{B}(M))$.

According to the above theorem, we introduce the concept of the dual matroid.

Definition 50 (dual matroid [27]). The matroid whose family of all bases is equal to $\operatorname{Com}(\mathscr{B}(M))$ is called the dual matroid of $M(U, \mathscr{I})$ and is denoted as $M^{*}$. In particular, if $\mathscr{I}=$ $\mathscr{I}\left(M^{*}\right), M$ is called an identically self-dual matroid.
Lemma 51 (see [27]). If $M=(U, I)$ is a matroid, then

$$
\mathscr{C}\left(M^{*}\right)=b(\mathscr{B}(M)), \quad b\left(\mathscr{C}\left(M^{*}\right)\right)=\mathscr{B}(M) .
$$

Now, based on the above results, a matroidal structure of covering-based rough sets will be constructed. Firstly, the concept of Eulerian covering is defined in the following definition.

Definition 52 (Eulerian covering). An Eulerian covering $\mathbf{C}$ is a covering of $U$ with the following properties:

(EC1) $\mathbf{C}$ is an antichain;

(EC2) there exist $K_{1}, K_{2}, \ldots, K_{m} \in \mathbf{C}$ such that $\left\{K_{1}, K_{2}, \ldots\right.$, $\left.K_{m}\right\}$ is a partition of $U$, where $m \in \mathbb{N}^{+}$;

(EC3) if $K, K^{\prime} \in \mathbf{C}, K \neq K^{\prime}$ and $k \in K \bigcap K^{\prime}$, then there exists $K^{\prime \prime} \in \mathrm{C}$ such that $K^{\prime \prime} \subseteq\left(K \bigcup K^{\prime}\right)-\{k\}$.

Example 53. Let $U=\{a, b, c, d, e, f\}$ and $\mathbf{C}=\{\{a, b, c\},\{a, b$, $d\},\{a, b, e\},\{a, b, f\},\{a, c, d\},\{a, c, e\},\{a, c, f\},\{a, d, e\},\{a, d$, $f\},\{a, e, f\},\{b, c, d\},\{b, c, e\},\{b, c, f\},\{b, d, e\},\{b, d, f\},\{b, e$, $f\},\{c, d, e\},\{c, d, f\},\{c, e, f\},\{d, e, f\}\}$. Clearly, $\mathbf{C}$ is a covering of $U$ and satisfies (EC1), (EC2), and (EC3) of Definition 52; then $\mathbf{C}$ is an Eulerian covering of $U$.

In fact, an Eulerian covering of a universe in compliance with the above definition satisfies the circuit axiom. In other words, it determines an Eulerian matroid.

Proposition 54. Let $\mathbf{C}$ be an Eulerian covering of $U$. Then $\mathbf{C}$ satisfies (C1), (C2), and (C3) of Theorem 17.

Proof. It is easy to prove this proposition by Theorem 17 and Definition 52.

Example 55 (continued from Example 53). As shown in Example 53, C is the family of circuits of an Eulerian matroid $M$. Clearly, $\mathscr{I}=\{X \subseteq U|| X \mid \leq 2\}$ and $\mathscr{B}(M)=\operatorname{Max}(\mathscr{I})=$ $\{B \subseteq U|| B \mid=2\}$.

Definition 56. Let $\mathrm{C}$ be an Eulerian covering on $U$. The Eulerian matroid whose circuit set is $\mathbf{C}$ is denoted by $M(\mathbf{C})$.

In fact, a partition of a universe is an Eulerian covering. Hence, a matroidal structure of classical rough sets is constructed in the following proposition.

Proposition 57. Let $R$ be an equivalence relation on $U$. Then there exists an Eulerian matroid $M(U / R)$ such that $\mathscr{C}(M(U / R))=U / R$

Proof. It is easy to prove that every partition of $U$ is an Eulerian covering. Hence, the proof of this proposition has already been finished.

Proposition 58. Let $R$ be an equivalence relation on $U$. Then

$$
\mathscr{B}\left(M^{*}\left(\frac{U}{R}\right)\right)=\operatorname{Min}\{X \subseteq U \mid \bar{R}(X)=U\} .
$$

Proof. For any $X \subseteq U$, it is obvious $(\forall A \in U / R, X \bigcap A \neq \emptyset) \Leftrightarrow$ $\bar{R}(X)=U$. Then according to Lemma 51, we know that 
$\mathscr{B}\left(M^{*}(U / R)\right)=b(\mathscr{C}(M(U / R)))=\operatorname{Min}\{X \subseteq U \mid \forall A \in U / R$, $X \bigcap A \neq \emptyset\}=\operatorname{Min}\{X \subseteq U \mid \bar{R}(X)=U\}$.

Example 59. Let $U=\{a, b, c, d, e, f\}$ and $U / R=\{\{a, e\}$, $\{b, d, f\},\{c\}\}$. Then $\mathscr{C}(M(U / R))=U / R$ is the family of circuits of an Eulerian matroid induced by $U / R$. In addition, suppose $M^{*}(U / R)$ is the dual matroid of $M(U / R)$. Then

$$
\begin{gathered}
\mathscr{B}\left(M^{*}\left(\frac{U}{R}\right)\right)=b\left(\frac{U}{R}\right)=\operatorname{Min}\{X \subseteq U \mid \bar{R}(X)=U\} \\
=\{\{a, b, c\},\{a, c, d\},\{a, c, f\}, \\
\{b, c, e\},\{c, d, e\},\{c, e, f\}\} .
\end{gathered}
$$

Proposition 60. Let $\mathrm{C}$ be an Eulerian covering of $U$ and $M^{*}(\mathbf{C})$ the dual matroid of $M(\mathbf{C})$. Then

$$
\mathscr{B}\left(M^{*}(\mathbf{C})\right)=\operatorname{Min}\{X \subseteq U \mid \overline{\mathbf{C}}(X)=\mathbf{C}\} .
$$

Proof. It is easy to prove this proposition by Lemma 51 and Proposition 33.

Example 61 (continued from Example 53). An Eulerian covering $\mathbf{C}$ of $U$ induces an Eulerian matroid $M(\mathbf{C})$. Suppose $M^{*}(\mathbf{C})$ is the dual matroid of $M(\mathbf{C})$. Then $\mathscr{B}\left(M^{*}(\mathbf{C})\right)=$ $b(\mathbf{C})=\operatorname{Min}\{X \subseteq U \mid \overline{\mathbf{C}}(X)=\mathbf{C}\}=\left\{B^{*} \subseteq U|| B^{*} \mid=4\right\}$.

\section{Conclusions}

In this paper, we studied one type of covering-based rough sets on Eulerian matroids. Via inducing a covering from the circuits of an Eulerian matroid, the covering-based approximation operators were represented from the viewpoint of Eulerian matroids. First, we studied the properties of the covering induced by an Eulerian matroid and the properties of the covering-based approximation operators through Eulerian matroids. Second, the relationships between two covering-based approximation spaces induced by two Eulerian matroids were explored. Finally, a matroidal structure of covering-based rough sets was constructed.

\section{Acknowledgments}

This work is in part supported by the National Science Foundation of China under Grant nos. 61170128, 61379049, and 61379089, the Natural Science Foundation of Fujian Province, China, under Grant no. 2012J01294, the Fujian Province Foundation of Higher Education under Grant no. JK2012028, and the Education Department of Fujian Province under Grant no. JA12222.

\section{References}

[1] Z. Pawlak, "Rough sets and fuzzy sets," Fuzzy Sets and Systems, vol. 17, no. 1, pp. 99-102, 1985.

[2] B. Sun, Z. Gong, and D. Chen, "Fuzzy rough set theory for the interval-valued fuzzy information systems," Information Sciences, vol. 178, no. 13, pp. 2794-2815, 2008.

[3] L. A. Zadeh, "Fuzzy sets," Information and Control, vol. 8, no. 3, pp. 338-353, 1965.
[4] Z. Pawlak, "Rough sets," International Journal of Computer and Information Sciences, vol. 11, no. 5, pp. 341-356, 1982.

[5] Z. Pawlak, Rough Sets: Theoretical Aspects of Reasoning about Data, Kluwer Academic, Boston, Mass, USA, 1991.

[6] F. Wang, "Outline of a computational theory for linguistic dynamic systems: toward computing with words," International Journal of Intelligent Control and Systems, vol. 2, pp. 211-224, 1998.

[7] L. A. Zadeh, "Fuzzy logic= computing with words", IEEE Transactions on Fuzzy Systems, vol. 4, no. 2, pp. 103-111, 1996.

[8] F. Wang, "Modeling, analysis and synthesis of linguistic dynamic systems: a computational theory," in Proceedings of the IEEE International Workshop on Architecture for Semiotic Modeling and Situation Control in Large Complex Systems, pp. 173178, 1995.

[9] Y. Yao and B. Yao, "Covering based rough set approximations," Information Sciences, vol. 200, pp. 91-107, 2012.

[10] Y. L. Zhang, J. Li, and W. Z. Wu, "On axiomatic characterizations of three pairs of covering based approximation operators," Information Sciences, vol. 180, no. 2, pp. 274-287, 2010.

[11] W. Zhu and F. Y. Wang, "Reduction and axiomization of covering generalized rough sets," Information Sciences, vol. 152, pp. 217-230, 2003.

[12] W. Zhu, "Relationship between generalized rough sets based on binary relation and covering," Information Sciences, vol. 179, no. 3, pp. 210-225, 2009.

[13] I. Couso and D. Dubois, "Rough sets, coverings and incomplete information," Fundamenta Informaticae, vol. 108, no. 3-4, pp. 223-247, 2011.

[14] T. Deng and Y. Chen, "On reduction of morphological covering rough sets," in Fuzzy Systems and Knowledge Discovery, vol. 4223 of Lecture Notes in Computer Science, pp. 266-275, Springer, Berlin, Germany, 2006.

[15] T. Feng, J. Mi, and W. Wu, "Covering-based generalized rough fuzzy sets," in Rough Set and Knowledge Technology, vol. 4062 of Lecture Notes in Computer Science, pp. 208-215, Springer, Berlin, Germany, 2006.

[16] T. J. Li, "Rough approximation operators in covering approximation spaces," in Rough Sets and Current Trends in Computing, vol. 4259 of Lecture Notes in Computer Science, pp. 174-182, Springer, Berlin, Germany, 2006.

[17] Q. Liu, F. Min, H. Cai, and G. Yang, "Converting decision tables," in Proceedings of the 11th World Congress of International Fuzzy Systems Association, pp. 1391-1396, 2005.

[18] H. Whitney, "On the abstract properties of linear dependence," American Journal of Mathematics, vol. 57, no. 3, pp. 509-533, 1935.

[19] J. Edmonds, "Matroids and the greedy algorithm," Mathematical Programming, vol. 1, pp. 127-136, 1971.

[20] E. Lawler, Combinatorial Optimization: Networks and Matroids, Dover Publications, Mineola, NY, USA, 2001.

[21] X. Li and S. Liu, "Matroidal approaches to rough sets via closure operators," International Journal of Approximate Reasoning, vol. 53, no. 4, pp. 513-527, 2012.

[22] Y. Liu, W. Zhu, and Y. Zhang, "Relationship between partion matrioid and rough set through k-rank matroid," Journal of Information and Computational Science, vol. 8, pp. 2151-2163, 2012.

[23] S. Wang, Q. Zhu, W. Zhu, and F. Min, "Matroidal structure of rough sets and its characterization to attribute reduction," Knowledge-Based Systems, vol. 36, pp. 155-161, 2012. 
[24] S. Wang, Q. Zhu, W. Zhu, and F. Min, "Quantitative analysis for covering-based rough sets through the upper approximation number," Information Sciences, vol. 220, pp. 483-491, 2013.

[25] B. Yang and W. Zhu, "Matroidal structure of generalized rough sets based on symmetric and transitive relations," in Proceedings of the 26th Annual IEEE Canadian Conference on Electrical and Computer Engineering (CCECE '13), pp. 1-5, Regina, Canada, May 2013.

[26] Y. Yao, "Constructive and algebraic methods of the theory of rough sets," Information Sciences, vol. 109, no. 1-4, pp. 21-47, 1998.

[27] H. Lai, Matroid Theory, Higher Education Press, Beijing, China, 2001. 


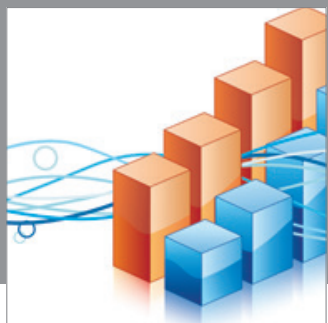

Advances in

Operations Research

mansans

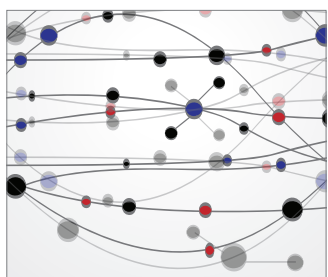

The Scientific World Journal
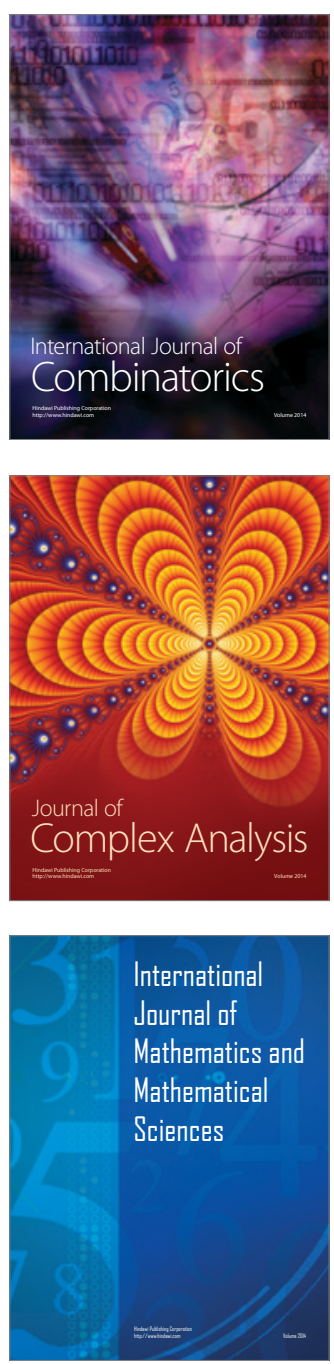
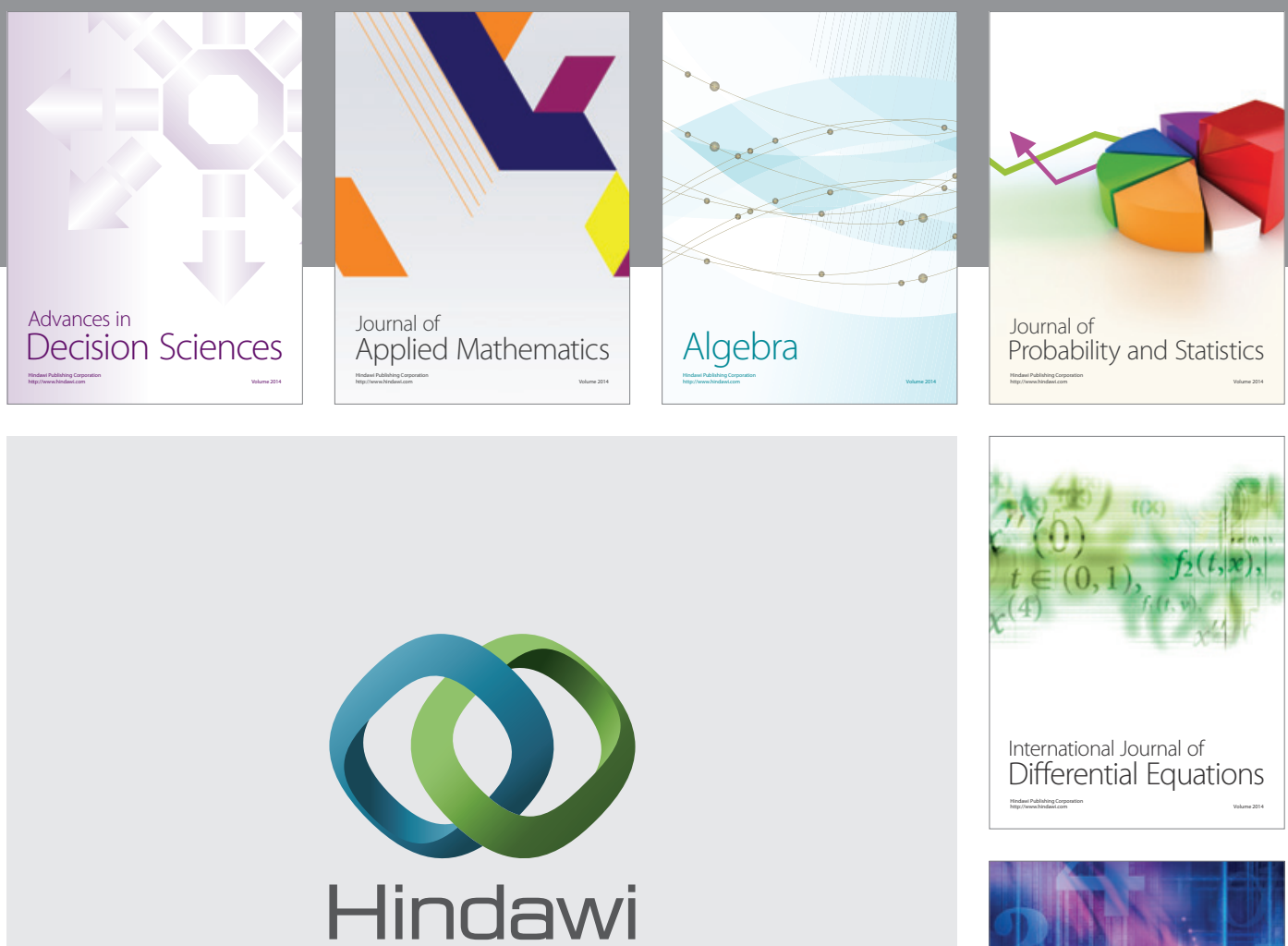

Submit your manuscripts at http://www.hindawi.com
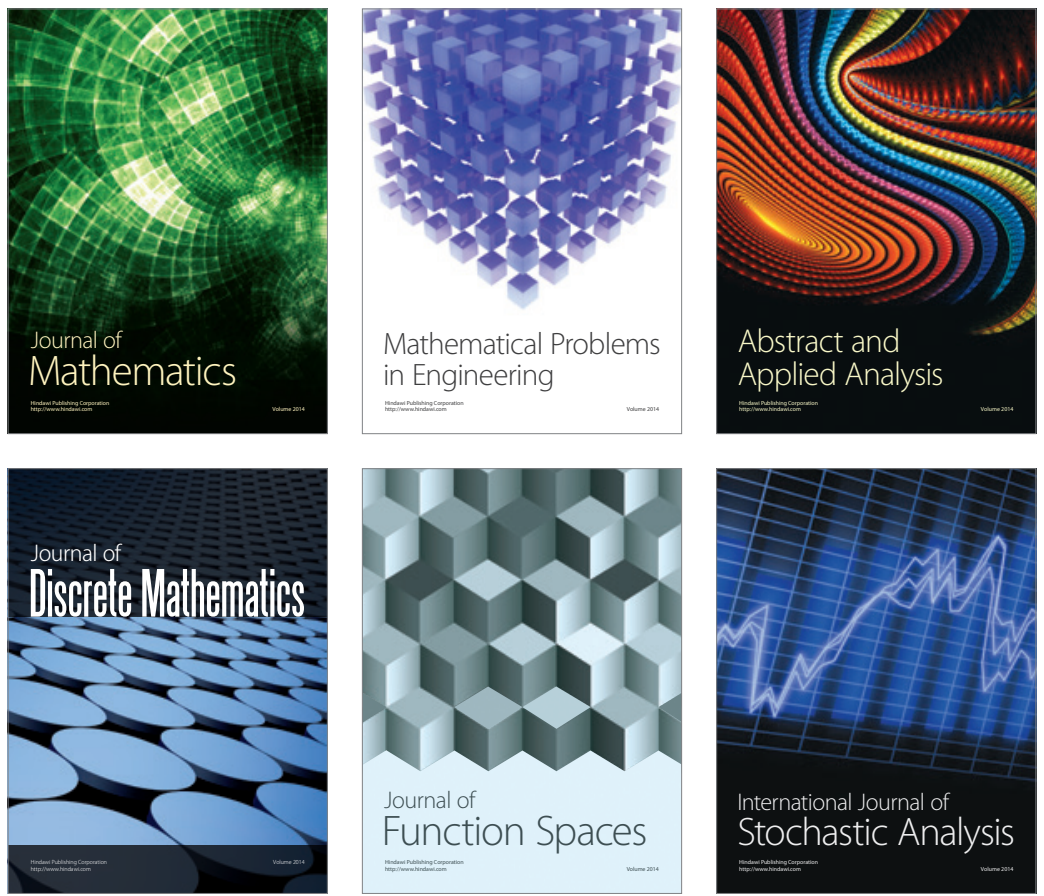

Journal of

Function Spaces

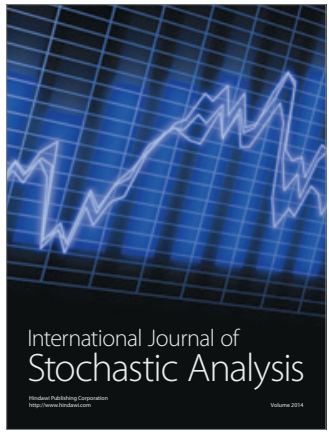

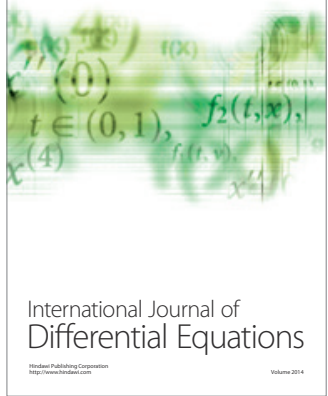
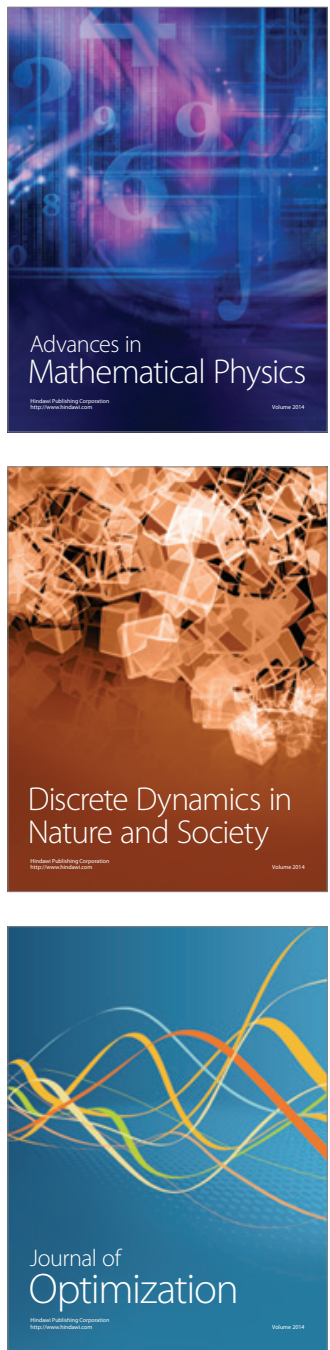